\title{
GESTIÓN DEL CUIDADO: VALORACIÓN Y CONOCIMIENTO DE ENFERMEROS(AS) DE UN HOSPITAL DE LA REGIÓN METROPOLITANA, CHILE
}

\section{CARE MANAGEMENT: NURSING KNOWLEDGE AND ASSESSMENT AT A METROPOLITAN REGION HOSPITAL, CHILE}

\author{
Jorge Valdebenito Pino* \\ ANA BARQUERO ** \\ María Elena Carreño ${ }^{* *}$
}

\begin{abstract}
RESUMEN
La unidad de gestión del cuidado (UGC) se enmarca en la reforma sanitaria chilena, adquiriendo importancia única para la profesión en el diseño, planificación, desarrollo y evaluación de los cuidados dentro de las instituciones sanitarias. El objetivo de este estudio fue describir la valoración y conocimiento que los(as) enfermeros(as) le otorgan a la UGC. Método: Estudio descriptivo, de corte transversal, el universo correspondió a 52 sujetos, a quienes se les aplicó un cuestionario estructurado, cuyas variables respondieron a las concepciones más significativas de la reglamentación actual de la UGC. Resultados: Se valoró positivamente la unidad, específicamente la relación comunicacional entre ésta y la actividad clínica; como necesidad se distinguió el reconocimiento de la institución a la UGC, y la formación en gestión del cuidado como parte fundamental en las competencias de los profesionales. Por otro lado, se observó que los individuos con menor experiencia laboral y de cargos de atención clínica corresponden a sujetos menos empoderados con las concepciones de la reglamentación de las UGC; por último las UGC en Chile son consideradas en mayor proporción como un ámbito de desarrollo gerencial, sin embargo surgieron opiniones respecto a la ampliación de la visión de estas unidades, considerándolas de manera más global, otorgándole un sentido y valor propio en la atención de enfermería. Conclusiones: El estudio logró describir una valoración positiva de las UGC en enfermeros de una institución sanitaria chilena, sin embargo se abren nuevas líneas de investigación, que se hace necesario profundizar a través de metodologías de investigación mixtas.
\end{abstract}

Palabras clave: Gestión del cuidado, enfermería, reforma sanitaria, administración hospitalaria, atención de enfermería.

\begin{abstract}
The care management unit (CMU) in Chile is framed within the public health reform, getting a unique importance for the profession in designing, planning, development and evaluation care within the health institutions. Objective: To describe the knowledge and the assessment that nurses put on the CMU. Method: Descriptive, cross-sectional study, with universe of 52 that answered a structured questionnaire, whose variables responded to the most significant concepts of the current regulation of CMU. Results: The unit was positively

\footnotetext{
*Enfermero. Máster@ en Ciencias de la Enfermería. Universidad de Huelva, España, Email: valdebenitopino@gmail.com ** Enfermera. Doctora en psicopedagogía, Docente Escuela de Enfermería Universidad de Huelva, España, Email: Barquero@ uhu.es

*** Licenciada en Enfermería, diplomada en Gestión en Salud, Email: mecarreno@hsoriente.cl
} 
considered, specifically the relationship between this unit and the clinical activity as need, the recognition of the institutional CMU and training in care management as a fundamental part in professional skills were recognized. Moreover, it was observed that individuals with less work experience and positions of clinical care, are less empowered subjects with conceptions of regulation of CMU; finally the CMU in Chile are considered in greater proportion as a field of management development, however emerged opinions on the extension of the vision of these units, considering them more globally, giving a sense and self-worth in nursing care. Conclusion: The study gets to describe a positive assessment of the CMU in health nurses in a Chilean institution, however new research lines are opened and, it is necessary to deepen through joint research methodologies.

Key words: Care management, nursing, health care reform, hospital administration, nursing care.

Fecha recepción: 22/11/12 Fecha aceptación: 19/08/14

\section{INTRODUCCIÓN}

La gestión del cuidado en la atención sanitaria Chilena se encuadra en la reforma del área a comienzos del 2007, cuyos objetivos pretenden mejorar la accesibilidad, cobertura y oportunidad a través del buen uso de recursos, gestión clínica y del cuidado, por medio del modelo integral de atención de salud. En este sentido, la mejora de los procesos clínicos asistenciales, en cuanto al rol de la enfermería, dependerá del empoderamiento y buen quehacer de las nuevas unidades de gestión del cuidado (UGC).

En Chile, la unidad de gestión del cuidado (UGC) corresponde a un elemento funcional en la gerencia de las instituciones, coordinando actividades en ámbitos propios de los "Cuidados de enfermería", éstas unidades reemplazan a las antiguas coordinaciones de enfermería, que poseían, hasta entonces, subordinación médica (1); por consiguiente estas unidades actualmente poseen un carácter normativo entregado exclusivamente al profesional enfermero.

En consecuencia con el tema, el concepto de unidades de gestión del cuidado (UGC) nace legalmente a través de un marco jurídico con la Ley $\mathrm{N}^{\circ} 19.536$ de 1997 en el libro $\mathrm{V}$ "Del ejercicio de la medicina y profesiones afines", Art. 113 inciso $4^{\circ}$, donde se señala que "los servicios profesionales de la enfermera comprenden la gestión del cuidado en lo relativo a promoción, mantención y restauración de la salud, la prevención de enfermedades o lesiones, y la ejecución de acciones derivadas del diagnóstico y tratamiento médico y el deber de velar por la mejor administración de los recursos de asistencia para el paciente" (2). A raíz de la incorporación en la Ley 19.937, comienza a aparecer la figura de las UGC dentro de las instituciones sanitarias; dado este mandato y considerando el nuevo rol de la enfermería, el Ministerio de Salud (MINSAL) pretende clarificar las concepciones precisas que esta unidad desarrolla, es así como, a través de la Norma Administrativa $\mathrm{N}^{\circ} 19$, desde el nivel central se aporta un documento normativo que, por un lado, viene a ratificar la conformación de las unidades y, por otro, le da una estructura organizacional a la gestión de enfermería en los organigramas hospitalarios.

De manera que, gestión de enfermería "implica gestionar la máxima cantidad del producto enfermero (el cuidado) en el conjunto de funciones que desarrollan los profesionales en el seno de las instituciones sanitarias, asistencia, docencia a usuarios y profesionales, participación en equipo de salud y desarrollo de los procesos de investigación para la mejora e innovación en la práctica enfermera" (3). En este sentido, éste modelo 
de gestión es la herramienta que promueven la estructura y organización de la actividad de la enfermería (4), a través de la reformulación de la distribución del trabajo y el reconocimiento del quehacer.

Por consiguiente, podemos acotar que el modelo de "Gestión del Cuidado" es un arquetipo de gestión, donde se incorpora el profesional enfermero, que requiere de herramientas administrativas inherentes a la profesión, actividades proporcionadas y coordinadas a partir de la gestora del cuidado, los cuales faciliten el planificar, organizar, dirigir y evaluar los recursos económicos, humanos y materiales, cuyo fin es proveer un cuidado integral dentro del ciclo vital a la persona, familia y comunidad; por lo tanto es necesario que la formación de enfermería posea conocimientos propios que faciliten el desarrollo de esta unidad, con el lenguaje enfermero y el proceso de enfermería como fundamento de su trabajo. La gestión del cuidado requiere una interacción activa y positiva entre la gestora del cuidado y los profesionales de enfermería, de forma que las experiencias y conocimientos sean entendidos como la oportunidad auténtica y única de desarrollo de la disciplina hacia su rol: el cuidado de la salud.

Estudios realizados en otros sistemas sanitarios, muestran que "las UGC estimulan el desarrollo profesional, permitiendo que el producto enfermero sea considerado por la organización como elemento de valor dentro del producto final (...) los profesionales opinan que ven una esperanza en la reivindicación del trabajo propio y una independencia real" (5), por tal razón, a partir de la reforma sanitaria se da inicio al cambio histórico para la disciplina de enfermería que debemos recoger, desarrollarlo y acentuar, haciéndolo un distintivo para la profesión. La nuevas unidades de gestión del cuidado requieren de un cambio de paradigma en la visión enfermera, un compromiso de todos en el desarrollo de esta unidad, con el fin de instruirse, participar y empoderarse de ella. Por eso se puntualiza que el conocimiento de los enfer- meros sobre la reglamentación de gestión del cuidado es "tan importante como tener a una buena gestora”. Sin ello, la gestión del cuidado sería una mera representación formal y estéril dentro de la organización hospitalaria, sin vinculación de las actividades propias de la ciencia de la enfermería y la asistencia hospitalaria. "En el proceso de gestión es necesario tener en cuenta también cuestiones de preocupación de responsabilidad personal y profesional. Esto, sin embargo, no puede atribuirse sólo a la enfermera jefe, como tal. Todos los profesionales, de este modo, se han comprometido a realizar cambios para promover la vida y la salud de las personas..." (5).

En la revisión de la literatura, se reveló que dentro del equipo de enfermería existió escaso conocimiento de las actividades y funciones que poseen las UGC, donde se percibió sólo como la organización del servicio a través de reglas, rutinas y horarios, desvinculándola de la atención directa de la enfermera. A partir de esto, se aprecia que existe una diferencia sustancial y real de la significancia de las unidades de gestión del cuidado, la cual correspondería no solo a la gestora, sino a la asistencia sanitaria a través de un mirar más amplio, de una mayor participación y contribución con el equipo (6). "El paso de la supervisión a la gestión de cuidados consiste en una reorientación hacia las tareas de cuidado, más énfasis en las funciones de gestión, asumir el control de la calidad asistencial, promover la aplicación de la metodología enfermera, realizar la evaluación del desempeño de los profesionales y apoyar su formación continua" (7).

En Chile, la conformación de las UGC ha sido un proceso progresivo y laborioso; aún se desconoce si el equipo de enfermería en Chile tiene los conocimientos necesarios para una adecuada implementación y cómo estos profesionales valoran estas unidades, cuáles son las implicancias ético-legales que trae consigo, en qué marco regulatorio se desenvuelve la gestión del cuidado. Desconocer es- 
tos aspectos va en desmedro principalmente de la profesión y la sanidad en Chile, según Rodríguez et al. (5): "Existen fases insalvables, la primera es conocer de los propios actores su percepción del cambio, a partir de esta cosmovisión construir estrategias hacia el cambio", por esta razón es que se pone a prueba a los actuales y nuevos profesionales, a familiarizarse sobre cuáles son las particularidades y pertenencias que el legislador faculta. Estudios realizados en el contexto español indican que los principales problemas de la puesta en marcha de la gestión de los cuidados radican en la falta de información y participación, por esto se promueve analizar los intereses de los enfermeros de atención clínica y de cargos directivos (7).

Desde aquí nace la necesidad de investigar si aquellos enfermeros, que participan en la atención directa de pacientes en las instituciones, saben ¿cuál es el conocimiento que tienen los enfermeros y cómo valoran la unidad de gestión del cuidado de enfermería? Nuestro objetivo es describir la valoración y conocimiento que los enfermeros(as) le entregan a la unidad de gestión del cuidado en un hospital de la Región Metropolitana, Chile, año 2011.

\section{MATERIAL Y MÉTODO}

El diseño de la investigación es observacional, descriptivo de corte transversal; el universo del estudio se constituyó por 58 individuos en actividad laboral permanente en un hospital de la Región Metropolitana de Chile, los cuales fueron ajustados a criterios de inclusión y exclusión.

Criterios de inclusión: Se incluyó a todos(as) los(as) enfermeros(as) de la institución, incluye cargos clínicos, supervisores(as) y encargados(as) de labores de gestión distinta de la gestión del cuidado, que cuenten con más de 6 meses de trabajo. Criterios de exclusión: enfermeros(as) que su contrato la- boral sea a medio tiempo (part-time), enfermeros(as) con menos de 6 meses de permanencia, enfermeros(as) que estén realizando algún reemplazo y enfermeras que trabajen en la unidad de gestión del cuidado, ya que estas últimas se espera que posean conocimiento de las UGC.

Para la recogida de datos, al no encontrar en nuestra búsqueda bibliográfica un instrumento validado que mida la valoración del conocimiento sobre la gestión del cuidado en Chile, se determinó la construcción de uno, donde se incluyan las concepciones del modelo de gestión del cuidado chileno. Para establecer la validez de contenido del instrumento fue sometido a prueba de jueces, respecto al área metodológica y área específica de la gestión de cuidado (incluyendo en ésta, académicos de distintas escuelas de Enfermería, gestores de cuidado, miembros del Colegio de Enfermeras); es así como la validez de criterio se abordó mediante un grupo de 13 expertos, quienes realizaron una depuración del instrumento, quedando constituido así un cuestionario estructurado con 2 partes: por un lado, 11 preguntas de los ámbitos sociodemográficos (edad, sexo, estado civil, entre otras), ámbitos de la trayectoria profesional (años de experiencia laboral, servicio donde trabaja, permanencia en la institución) y ámbitos formación profesional (universidad donde estudió, estudios de postítulo, estudios sobre gestión del cuidado) y, por otro lado, tres dimensiones (ético-legal, clínico-asistencial, gerencial-administrativo), los cuales poseen 27 preguntas con respuestas tipo Likert, totalmente de acuerdo a totalmente en desacuerdo (Anexo 1). Además, se realizó un pilotaje a 10 enfermeros(as) distintos al universo, donde sólo se modificó aspectos de género relacionados al cuestionario.

Para fiabilidad de la investigación, se controló mediante la recolección de datos durante los meses de julio a septiembre del año 2011, en horario hábil, de forma estandarizada, previa coordinación con la UGC de la 
institución; la fiabilidad interna por medio del cálculo de Alfa de Cronbach global de 0,849 para los 27 enunciados del cuestionario "Gestión del cuidado -Chile" (Anexo 1).

Desde el punto de vista ético, la investigación fue sometida al Comité de Ética de la institución, cuyo posterior análisis y evaluación del instrumento permitió fuera aceptada para su aplicación, asimismo la participación de los individuos fue de forma voluntaria y anónima, complementando un consentimiento informado adjunto a cada cuestionario. Los datos obtenidos fueron procesados mediante el software estadístico Statistical Package for the Social Sciences (17.0), donde se realizó análisis univariado de las distintas variables del cuestionario, obteniendo medi- das de tendencias central, frecuencias y porcentajes.

\section{RESULTADOS}

De la población total de enfermeros(as), la participación alcanzó a un 89,6\% correspondiente a 52 individuos; según la distribución por género: $14 \%$ de género masculino y $86 \%$ femenino. El rango de edad con mayor número de profesionales fue de 31-40 años, $51,9 \%$ de los individuos tenía menos de 40 años y sólo un 13,5\% más de 50 y menos de 25 años (Tabla 1).

Tabla 1. Caracterización sociodemográfica, formación y experiencia profesional.

\begin{tabular}{lcc}
\hline & n & $\%$ \\
\hline Sexo & 7 & 14 \\
Masculino & 45 & 86 \\
Femenino & & \\
Edad & 3 & 5,8 \\
$<25$ años & 8 & 15,4 \\
$26-30$ & 27 & 51,9 \\
$31-40$ & 10 & 19,2 \\
$41-50$ & 4 & 7,7 \\
$>50$ años & & \\
Distribución según cargos en la institución & 38 & 73 \\
Enfermeros/as clínicos/as por turno & 7 & 13 \\
Enfermeros/as supervisores/as & 4 & 8 \\
Enfermeros/as clínicos/as diurno & 3 & 6 \\
Enfermeros/as en labor de gestión & & \\
Área de formación de postítulo & 18 & 34 \\
Infecciones Intrahospitalarias & 10 & 19,1 \\
Medicina intensiva & 9 & 17,1 \\
Calidad & 9 & 17 \\
Gestión y administración & 7 & 12,9 \\
Otras & & \\
Distribución formación académica de postítulo & 4 & 7,7 \\
Magíster & 24 & 46,2 \\
Diplomado & 19 & 36,5 \\
Cursos de capacitación & 5 & 9,6 \\
Otros & &
\end{tabular}


Los cargos de supervisión y gestión lo realizaban en mayor proporción el género masculino. Por otro lado, según los años de permanencia, el 56\% tenía más de 8 años de trabajo en la institución.

Respecto al cargo desempeñado, en los cargos de supervisión el 71,5\% tenía más de 8 años de trabajo en la institución. Cabe mencionar que de las enfermeras con labor de gestión el 66,7\% tenían entre 6 meses y 3 años en la institución. Por otra parte las enfermeras clínicas se distribuyeron en todas los tramos de años de trabajo en la institución (Gráfico 1).

Gráfico 1. Distibución por años de trabajo en la institución vs cargo desempeñado.

\begin{tabular}{|c|c|c|c|c|c|c|c|c|}
\hline $\begin{array}{r}18 \\
16 \\
14 \\
12 \\
10 \\
8 \\
6 \\
4 \\
2 \\
0\end{array}$ & I & - & H & & 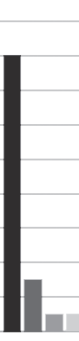 & & W & 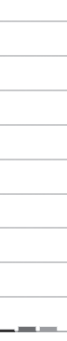 \\
\hline & $\begin{array}{c}6 \\
\text { meses-3 } \\
\text { años }\end{array}$ & & $\begin{array}{c}\text { 4-7 } \\
\text { años }\end{array}$ & & $\begin{array}{l}\text { 8-10 } \\
\text { años }\end{array}$ & & $\begin{array}{l}\text { Más de } \\
10 \text { años }\end{array}$ & \\
\hline E.U. clínica por turnos & 7 & 0,18 & 10 & 0,26 & 16 & 0,42 & 5 & 0,13 \\
\hline E.U. supervisora & 1 & 0,14 & 1 & 0,14 & 3 & 0,43 & 2 & 0,29 \\
\hline E.U. clínica diurna & 0 & 0 & 2 & 0,50 & 1 & 0,25 & 1 & 0,25 \\
\hline E.U. de gestión & 2 & 0,67 & 0 & 0 & 1 & 0,33 & 0 & 0 \\
\hline
\end{tabular}

Según la formación de postítulo, el grupo de enfermeros clínicos ostentaba más formación de postítulo (cursos de capacitación y diplomados) que los enfermeros supervisores y gestores; particularmente solo un $57,1 \%$ de las enfermeras supervisoras han realizado formación en gestión y administración, y un 33\% de las enfermeras en cargos de gestión poseen estudios de calidad. Si se analiza desde la edad de los sujetos, los participantes que tienen entre 31 y 40 años son los que más habían realizado cursos de formación de posgrado $(51,9 \%)$, en comparación con los de menos de 25 años con solo un 5,8\%. De los participantes con magíster, el $100 \%$ tenía entre 31 y 50 años. Sobre los 31 años, el $78,8 \%$ de los individuos ha realizado alguna formación de posgrado/postítulo (Gráfico
2). Por último, respecto a la formación específica en gestión del cuidado se observó que solo $4(7,7 \%)$ profesionales, del total de la población, posee este tipo de formación. Las áreas de formación de postítulo se observan en Tabla 1.

Al consultar sobre aspectos propios de la gestión del cuidado (Tabla 2): en la pregunta "La UGC facilitan el desempeño de la disciplina de enfermería", se obtuvo una mediana de 4,5, que corresponde a las respuestas totalmente de acuerdo y de acuerdo. Asimismo el 78,8\% de los participantes estuvo a favor que la UGC participa en el desarrollo de la disciplina de enfermería; cabe destacar que los profesionales con menos de 7 años de trabajo en la institución (21,2\%) no estuvieron de acuerdo con el enunciado anterior. 
Gráfico 2. Distribución de formación de postítulo según años de trabajo.

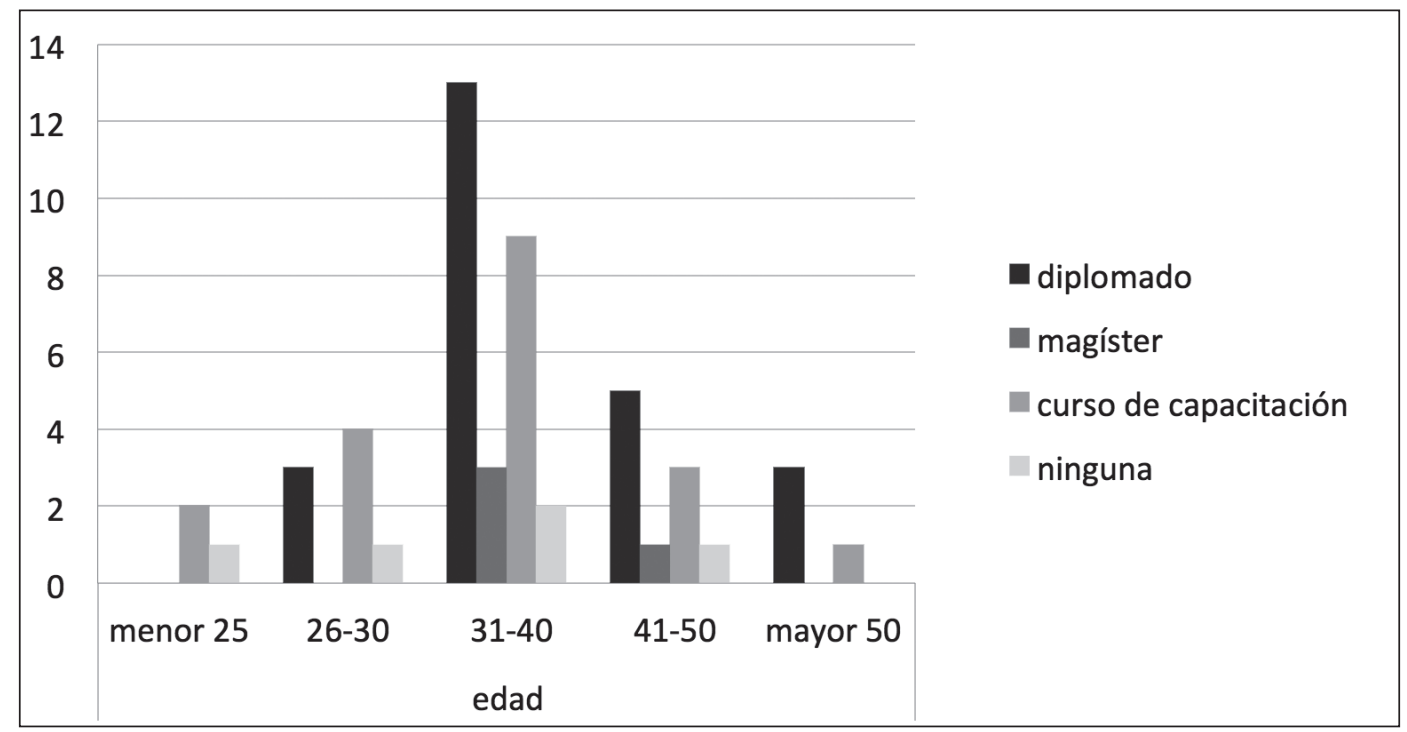

Tabla 2. Valoración y conocimiento respecto a la gestión del cuidado en enfermeras(os) de un hospital de la Región Metropolitana, Chile. 2011.

N válido Media Mediana Moda DE

\section{Respecto a la gestión del cuidado}

¿Las UGC facilitan el desempeño de la disciplina de enfermería?

$\begin{array}{lllll}52 & 3,98 & 4,5 & 5 & 1,379\end{array}$

¿Considera que la GC trae consigo implicancias ético-legales?

$\begin{array}{lllll}52 & 4,4 & 4 & 4 & 0,603\end{array}$

¿Conoce las normativas relacionadas a la gestión del cuidado?

$52 \quad 4,1 \quad 4 \quad 4 \quad 0,955$

\section{La gestión del cuidado, ¿Cómo la considera Ud.?}

Actividad sólo realizada por el gestor, por lo tanto no puedo realizar GC desde mi puesto de trabajo

Actividad realizada por las enfermeras en conjunto

Actividad de trabajo en conjunto entre equipo y gestora del cuidado

Otro profesional realiza gestión del cuidado

¿La actividad principal de la UGC es?

Planificar, organización, dirección y control de los cuidados integrales de enfermería

Diseñar, realizar y evaluar protocolos o guías clínicas

$52 \quad 1,54 \quad 1 \quad 110,851$

Corresponde a la instauración de un modelo teórico de enfermería para las actividades diarias

Facilitar el empoderamiento de la enfermería en la institución

$\begin{array}{lllll}52 & 4,5 & 5 & 5 & 0,7\end{array}$

$52 \quad 4,65 \quad 5 \quad 5 \quad 0,556$

\begin{tabular}{lllll}
52 & 2,4 & 2 & 1 & 1,587 \\
\hline
\end{tabular}

\section{¿Cómo conceptualiza o concibe Ud. la gestión del cuidado en su unidad?}

Esta actividad sólo le corresponde al gestor(a) del cuidado, por lo tanto es imposible realizar gestión desde mi puesto de trabajo

\begin{tabular}{lllll}
52 & 4,71 & 5 & 5 & 0,457 \\
52 & 4,23 & 4 & 4 & 0,877 \\
52 & 4,21 & 4 & 5 & 0,997 \\
52 & 4,5 & 5 & 5 & 0,98 \\
\hline
\end{tabular}

Se realiza siguiendo los lineamientos desde la UGC, facilitando la retroalimentación de la actividad clínica asistencial

$\begin{array}{lllll}52 & 1,58 & 1 & 1 & 0,997\end{array}$

Como una actividad global desde el gestor hasta las bases (enfermeros clínicos)

$\begin{array}{lllll}52 & 4,58 & 5 & 5 & 0,696\end{array}$

$\begin{array}{lllll}52 & 4,75 & 5 & 5 & 0,556\end{array}$


Continuación Tabla 2

¿Cómo implementa Ud. la gestión del cuidado en su unidad?

Poniendo en marcha protocolos, registros y notas de enfermería de la institución

Participando activamente en las actividades de enfermería, utilizando como base las teorías propuestas

Facilitando la retroalimentación entre la actividad clínica y la supervisión de enfermería

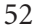

$\begin{array}{lllll}52 & 2,5 & 1 & 5 & 1,265 \\ 52 & 4,23 & 1 & 5 & 0,996\end{array}$

$52 \quad 4,6 \quad 1 \quad 5 \quad 0,566$

¿Qué ámbitos considera Ud. que comprende la gestión del cuidado?

\begin{tabular}{|c|c|c|c|c|c|}
\hline Ámbito clínico asistencial & 52 & 4,77 & 5 & 5 & 0,546 \\
\hline Ámbito administrativo & 52 & 4,62 & 5 & 5 & 0,491 \\
\hline Ámbito docente/investigación & 52 & 4,46 & 5 & 5 & 0,699 \\
\hline Ámbito gerencial & 52 & 4,6 & 5 & 5 & 0,495 \\
\hline Ámbito legal & 52 & 3,8 & 4 & 4 & 0,693 \\
\hline \multicolumn{6}{|c|}{ ¿Qué elementos considera Ud. como pilares fundamentales para que la UGC se lleve a cabo en su unidad? } \\
\hline Investigación en enfermería & 52 & 4,25 & 4,5 & 5 & 0,968 \\
\hline Formación continua en GC & 52 & 4,69 & 5 & 5 & 0,673 \\
\hline Formación académica en GC desde el pregrado & 52 & 4,48 & 5 & 5 & 0,7 \\
\hline Participación activa de los enfermeros en la atención integral al usuario & 52 & 4,62 & 5 & 5 & 0,491 \\
\hline Reconocimiento de la institución sobre la GC de enfermería & 52 & 4,83 & 5 & 5 & 0,382 \\
\hline
\end{tabular}

El 98\% de los individuos respondió que están de acuerdo y totalmente de acuerdo, que la UGC trae implicancias ético-legales para la profesión; igualmente el $86,5 \%$ respondió favorablemente hacia el conocimiento de las normativas relacionadas a la gestión del cuidado. Solo los de menor edad (menos de 30 años) se muestran indiferentes a la consulta anterior.

Respecto de la aseveración "Que la actividad de GC solo la realiza el gestor, por lo tanto no puedo realizar GC desde mi puesto de trabajo", la media fue 1,54 , es decir se encuentra entre totalmente en desacuerdo y desacuerdo, con un concluyente rechazo, es decir un $94,2 \%$ estuvo en desacuerdo (opciones 1 y 2 ).

Similar resultado se encontró frente a la aseveración "Otro profesional realiza gestión del cuidado", el 96,1\% respondió en desacuerdo y totalmente en desacuerdo, con una media de 2,40, los individuos que tenían más de 8 años de permanencia en la institución presentaron mayor rechazo $(68,9 \%)$ que quienes tenían menos de 7 años de perma- nencia $(43,5 \%)$.

A propósito de la pregunta si la "actividad de gestión del cuidado es realizada por las enfermeras en conjunto", y si "la actividad de trabajo de gestión del cuidado se realiza en conjunto entre equipo y gestora del cuidado", las respuestas fueron favorables para ambas preguntas, el 92,2\% de los individuos respondió totalmente de acuerdo y en acuerdo para la primera, con una media de 4,50, y el $96,1 \%$ para la segunda respuesta, con una media de 4,65.

Todos los participantes respondieron de acuerdo y totalmente de acuerdo a la pregunta de "La actividad principal de la UGC es planificar, organización, dirección y control de los cuidados integrales de enfermería", la media fue 4,71. Por otra parte, es importante destacar que el 92,2\% estuvo de acuerdo y totalmente de acuerdo que la "UGC facilita el empoderamiento de la enfermería en la institución", con una media de 4,50 (DE 0,98).

Desde el punto de vista de la metodología, la consulta sobre si la UGC "Corresponde a la instauración de un modelo teórico de enfer- 
mería para las actividades diarias", el 86,6\% consideró que está de acuerdo y totalmente de acuerdo, sólo un 13,5 estuvo en desacuerdo, y que correspondió a grupo específico mayoritariamente enfermeros clínicos por turnos $(90,2 \%)$ y que tenían entre 4 a 7 años en la institución (9,9\%).

A destacar que el $100 \%$ de los individuos estuvieron totalmente de acuerdo y en acuerdo, que la "gestión del cuidado se realiza siguiendo los lineamientos desde la unidad de gestión", para esta aseveración se obtuvo una media de 4,58. Respecto a la vinculación de la gestión del cuidado, "La gestión del cuidado es una actividad global desde el gestor hacia las bases", las respuestas tuvieron una media de 4,75 , lo que indica que las respuestas fueron favorables.

En relación a la aseveración cuáles son los ámbitos de enfermería en que se desenvuelve la UGC, existieron dos alternativas, donde el total de los participantes responde favorablemente, éstas son "ámbito gerencial y de administración". Por otro lado, solo un $3,8 \%$ consideraron el ámbito legal como una condición aplicable a la GC, quienes estuvieron en desacuerdo pertenecían a unidades clínicas de mediana y baja complejidad, a diferencia de las unidades ambulatorias o de gestión; cabe mencionar además que fueron profesionales con menos 7 años de trabajo en la institución. Asimismo, en el "ámbito docente/investigación" un $92,3 \%$ estuvo de acuerdo; se observó que los individuos sin formación en posgrados rechazaron esta alternativa como un ámbito de la GC.

Respecto de la pregunta "qué elementos considera Ud. como pilar fundamental para que la UGC se lleve a cabo en su unidad", existió una unanimidad en responder favorablemente todas las aseveraciones, las medias estuvieron entre 4,25 y 4,83 . La que mayor variabilidad mostró fue "investigación de enfermería", con una desviación estándar de 0,968 . En las respuestas de "Participación activa de los enfermeros en la atención integral al usuario" y "Reconocimiento de la institu- ción sobre la gestión del cuidado de enfermería", ambas tuvieron afirmación positiva con una desviación estándar menor 0,491 para la primera y 0,382 para la segunda.

Es importante mencionar que el grupo de participantes que poseían formación en gestión del cuidado respondieron en mayor proporción a enunciados acordes a los conceptos de gestión del cuidado citados en el marco conceptual, es decir poseen conocimiento teórico y práctico de lo que corresponde a las UGC.

\section{DISCUSIÓN Y CONCLUSIÓN}

Respecto a la caracterización de los participantes, podemos concluir que existió amplia formación de postítulos en enfermeros clínicos, en comparación a supervisoras y gestoras, contrario a lo declarado por Cabellos Vargas y Gorritti Siappo, donde destacan que dentro de los problemas de enfermeras asistenciales está la baja formación continuada (8); sin embargo, no concordando con Gómez-Torres et al., quienes afirman que dentro de la estrategia gerencial de una institución los requisitos que se establecen para los cargos directivos y gerenciales deben ser acordes a su formación (9); en el caso de nuestro grupo de estudio se observa una potencial herramienta en la mejora de los procesos de empoderamiento en la gestión desde los profesionales de atención clínica, pero que, sin embargo, abre una brecha sobre la necesidad de la continuidad de la formación en gestoras.

Respecto al conocimiento específico de los enfermeros sobre la "gestión del cuidado", se observó escasa formación en el área; de quienes sí se capacitaron, podría ahondarse en futuros estudios de tipo cualitativo para conocer cuáles fueron las motivaciones o intereses que llevaron a estos profesionales a formarse en esta área; este estudio solo puede aportar que los participantes, de acuerdo a 
su "cargo y edad", responden a una necesidad profesional para aumentar el conocimiento del nuevo modelo de gestión de enfermería según concepciones que trae la reforma. Por cierto, hay que indicar que el acceso a este tipo de formación de posgrado aún es limitado en nuestro país, y no es considerado como relevante en el currículo de posgrado, ni requerido como competencia mínima al momento de concursar por un cargo, dato contrastado en nuestro grupo de estudio, quienes prefieren formación en otras áreas más clínicas (infecciones asociadas a atención de salud, calidad, oncología, atención prehospitalaria, etc.).

Sobre la "valoración de la unidad de gestión del cuidado en su institución", los aspectos sobre la comunicación ente gestora y enfermeros asistenciales fueron valorados positivamente, es decir se da significación a la retroalimentación de la información clínica, vista ésta desde un terreno actitudinal/ comunicacional, corresponde a un aspecto positivo para la UGC. En esta línea, en el estudio de Faustino et al., se observó que la UGC fue una entidad reconocida como facilitadora del trabajo diario (6), así como también la construcción de una identidad profesional por el reconocimiento de la actividad realizada (7).

Al momento de definir la "designación del rol de gestor del cuidado", existió una correcta comprensión, la responsabilidad de la gestión no sólo recayó en la gestora, sino se consideró un trabajo entre gestora y enfermeras clínicas como parte del proceso, esto concuerda con lo expresado por los distintos autores, donde indican que es necesario un trabajo en conjunto de los enfermeros asistenciales para una buena gestión $(3,5,10$, 11).

En la pregunta que hace referencia a la participación de otro profesional sanitario en la UGC, ésta es una temática que causa controversia y que, por supuesto, requiere un análisis especial, pero que concierne a la in- corporación de otros gremios a la gestión del cuidado. En los participantes de nuestro estudio esta pregunta fue la que presentó mayor variabilidad "tanto a favor como en contra", específicamente en profesionales con menos años de trabajo, en cambio aquellos profesionales con más años de trabajo se mostraron en desacuerdo, por consiguiente se deduce que en estos últimos, existe mayor empoderamiento de su rol y significación con la unidad, quizás a través de una lucha continua hacia la autonomía de la profesión. Respecto a la UGC en Chile, Milos et al. aclaran que "la gestión del cuidado es intransferible en cuanto se requiere de un criterio profesional que, desde su planificación hasta su ejecución, responda a estándares éticos, científico-técnicos y jurídicos, que sólo se alcanzan con una formación superior impregnada de la tradición histórica de la enfermería" (12).

Al consultar sobre una definición de la GC y su ámbito de acción, los participantes están familiarizados uniformemente con los conceptos del proceso administrativo (organización, planificación, control y evaluación) y lo vinculan a la unidad, coincidiendo con lo descrito por Milos et al. (12), Zárate Grajales (13), Estefo y Paravic (14) y Gómez-Torres et al. (9); esta adecuación al proceso administrativo, consideramos que puede ser vinculante a la formación profesional y la asociación de GC solo al concepto administrativo.

El uso de herramientas para las prácticas de la gestión del cuidado corresponde a una actividad importante en la disciplina, por ejemplo su constructo metodológico y formativo al momento de abordar un tema en específico $(7,13,15)$. Al consultar a los participantes "cómo implementaría la GC en su unidad", el uso del proceso de enfermería (teorías de enfermería), un 21,2\% de los participantes responde desfavorablemente que sea una actividad que favorece la GC; en este sentido, Thorfehn et al. señala: "Resalta que existe gran diferencia entre la teoría y la práctica" (15). Este rechazo del uso 
de teorías estuvo presente en individuos con menos años de trabajo de institución (menos de 3 años) y que poseen cargos clínicos, por lo que sería útil, para la gestora y los otros profesionales vinculados a fortalecer directamente la gestión del cuidado, analizar cuáles son los obstáculos que incitan a enfermeros a manifestarse en contra del uso de teorías o qué relación existe con la formación profesional y el uso de herramientas formativas en la práctica, considerando el nivel de uso que existe desde aprendizaje académico.

Por otro lado, el trabajar en el diseño de protocolos o guías clínicas para la actividad asistencial, casi un 1/3 desestimó la importancia de esta actividad en la GC; respuesta distinta a la encontrada en el estudio sobre el uso "Instrumentos administrativos: percepciones de enfermeros de un hospital universitario" (16), donde los participantes reconocen en su mayoría la necesidad de crear y utilizar herramientas para la normalización y organización de las actividades en la institución (16).

Respecto a la identificación en la UGC, los participantes del presente estudio perciben esta necesidad de reconocerse en la unidad; así también que la organización, "más que la unidad misma", reconozca a los enfermeros su actividad de gestión del cuidado como propia, precisamente coincide quienes respondieron afirmativamente son participantes sujetos con más años de trabajo en la institución. En el estudio "De gestoras de recursos a gestoras de cuidados: opiniones y expectativas de las supervisoras sobre el nuevo rol profesional" (7), se reconoce que una de las dificultades hacia la implementación de la gestión del cuidado enfermería es el escaso reconocimiento de las tareas desarrolladas por las/os profesionales, lo que dificultaría su desarrollo. Asimismo. Rodríguez et al. (5) describe "que los profesionales opinan que la unidad de gestión del cuidado es un proyecto de mejora general, que puede estimular el desarrollo, permitiendo que el producto en- fermera sea considerado por la organización".

En el análisis de "los elementos que considera como pilar fundamental para que la gestión de cuidados se lleve en su unidad", la formación especializada en gestión del cuidado y la investigación en enfermería fueron los contenidos que presentaron respuestas para un mayor análisis. Del primero podremos observar que existió necesidad de formación en GC entre todos los profesionales consultados, Prieto-Rodríguez et al. (7) concuerdan con este enunciado; del segundo, que correspondió a la investigación como elemento considerado significativo, especialmente en individuos con formación de posgrado; en este sentido otras investigaciones concuerdan con las opiniones de nuestros participantes. Seguel y Paravic expresan "Los profesionales de enfermería bajo la UGC pueden tener protagonismo en investigación, relacionados con la gestión de servicios y los cuidados para con los pacientes" (17).

Para la formación en GC, una alternativa válida es incluirla en las competencias adquiridas desde pregrado, considerando la gestión del cuidado desde su ámbito jurídico hasta su trabajo asistencial, áreas que pueden ser evaluadas en el Examen Nacional de Enfermería, actualmente en fase de pilotaje.

En relación a los ámbitos que comprende la gestión del cuidado, lo administrativo y gerencial fueron considerados por todos los participantes como fundamentales, por lo tanto homologan la GC como gerencia y administración, a diferencia de otros estudios (18) donde las áreas gerencial y administrativa estuvieron valoradas en cuarto lugar por los enfermeros. En segundo lugar fue el asistencial, ámbito providencial ya que la actividad clínica es el reflejo palpable de la gestión de los cuidados (18). Se valoró en tercer lugar el ámbito docente-investigación. Harrison et al. (19) expresan que la investigación es función ineludible de la profesional de enfermería, siendo poco valorada por las instituciones de salud, y poco exigida en 
el actuar de enfermería, y más aún, colocada en último plano por el mismo profesional de enfermería. En último lugar se valoró el ámbito ético-legal; precisamente quienes mayormente le designaron menor importancia a este ámbito corresponden a participantes más jóvenes y de cargos de atención directa; aspecto a subrayar y que debe mirarse más a fondo, considerando que la reglamentación de las unidades, así como también las consideraciones que conlleva la implementación de una unidad de gestión del cuidado, adquieren aspectos legales y éticos que deben ser conocidos y ponderados. En este sentido Chailán y Conchall (20) analizan qué la experticia profesional a través de la experiencia pone en juego nuevos conocimientos éticos, donde el experto debe tomar decisiones frecuentemente y hacerse cargo de estas decisiones, y en este sentido los nuevos participantes tienen menos reconocimiento a aspectos relacionados con las decisiones clínicas y legales. Este ámbito es un punto clave para indagar en futuras investigaciones, en la línea de medición de competencias específicas, ya que la formación profesional está impregnada de la ética, por lo tanto establecer este requisito sería fundamental en la organización, considerando lo que Sánchez (21) señala, que aproximadamente alrededor de un 30\% de los casos tratados en el ámbito sanitario conllevan la resolución de un problema ético importante.

Se considera que un hallazgo para el estudio es la valoración de este ámbito, lo que determinaría un punto de partida para analizar qué formación tienen estos profesionales desde el pregrado, precisamente de su escuela de Enfermería: ¿se les imparte la ética relacionada a la complejidad de la gestión del cuidado?, ¿qué exigencias les solicita el hospital a la hora de utilizar los conocimientos en la atención clínica?, ¿existe participación o qué papel juegan las enfermeras en la toma de decisiones en cada actividad?, entre otras preguntas. Se abren, entonces, nuevas líneas de investigación.

Al respecto, los participantes con "formación en gestión del cuidado" destacan la identidad de la gestión según la actividad gerencial/administrativa, el empoderamiento del proceso administrativo y el uso de protocolos, entre otros. Asimismo, fue posible observar que en estos individuos existe una clara conceptualización de la UGC, reconociendo el rol del profesional de enfermería, las implicancias de la actividad de la unidad, así como también el apoyo profesional que la unidad puede facilitar a la enfermería.

Se concluye que la valoración de la UGC aún se distingue como una unidad mayoritariamente gerencial y administrativa, enmarcada en la definición del proceso administrativo, donde la participación fue considerada útil por todos los participantes del estudio y no solo el gestor. Como un punto a favor de la UGC fue identificar que la retroalimentación de la información clínica es clave en la puesta en marcha de esta unidad. Por el contrario, se visualizó que el grupo con menos años de trabajo y de menor edad cronológica fue el que se manifestó negativamente a la cultura y éxito de la unidad, como por ejemplo: la práctica asistencial bajo el desconocimiento de normativas, no consideración de aspectos éticos legales, entre otros.

Se estableció que existen áreas específicas no valoradas por la totalidad y que aún queda por trabajar, como la investigación en enfermería y el uso de herramientas propias (teorías de enfermería) para el trabajo clínico. Respecto al conocimiento, se identificó que existe una necesidad evidente de formación en gestión del cuidado y que ésta podría favorecer las concepciones correctas de la reglamentación para evitar comprenderla mayoritariamente de forma gerencial, sino que verla desde una esfera más amplia, con participación y empoderamiento de todos los enfermeros, con el fin de darle una importancia real a la gerencia del cuidado de enfermería en Chile. 


\section{REFERENCIAS}

1. Caballero E, Becerra R, Hullin C. Proceso de enfermería e informática para la gestión del cuidado. Santiago, Chile: Mediterráneo; 2010.

2. Código Sanitario. Decreto con Fuerza de Ley No 725 , actualizado a julio de 2000. Libro V, Artículo 113, inciso cuarto. [citado 12 enero 2013]. Disponible en: http://www.leychile.cl/Navegar?idNor$\mathrm{ma}=81502 \&$ buscar $=$ CODIGO + SANITARIO+1953

3. Asenjo Sebastián MA. Gestión diaria del hospital. Barcelona: Masson; 2000.

4. Milos P, Bórquez B, Larrain AI. La "Gestión del Cuidado" en la Legislación Chilena: Interpretación y Alcance. Cienc. enferm. 2010; XVI (1):17-29.

5. Rodríguez Bello J, López Sánchez A, Martínez Mora M, Balsera Balsera M, Gómez Fernández C, Jiménez Zarco C, et al. Unidades de gestión de cuidados: ¿qué opinan los profesionales de su implantación? Revista Rol de Enfermería. 2002; 25(3): 210-215.

6. Faustino AM, Reis PED, Jesus CAC, Moura EF, Medeiros KC, Amorin MAN. Models management of nursing care: qualitative study. Online braz.j.nurs. [Internet]. 2010 [citado 1 abril 2014]; 9(1). Disponible en: http://www.objnursing.uff.br/index.php/nursing/article/ view/j.1676-4285.2010.2843

7. Prieto-Rodríguez MA, Suess A, March-Cerdá JC. De gestoras de recursos a gestoras de cuidados: opiniones y expectativas de las supervisoras sobre el nuevo rol profesional. Enfermería Clínica. 2005;15(6):314-320.

8. Cabellos Vargas D, Gorritti Siappo C. Percepción de las enfermeras asistenciales en la aplicación del método de supervisión del departamento de enfermería. Trujillo, 2010. In Crescendo [Internet]. 2011 ene-jun [citado 1 abril 2014]; 2 (1):199-209. Disponible en: http://re- vistas.concytec.gob.pe/scielo.php?pi$\mathrm{d}=$ S2222-30612011000100016\&script $=s$ ci_arttext

9. Gómez-Torres D, Ángeles-Ávila G, Martínez-Garduño MD. Modelo de dirección de hospitales por enfermeras. Aquichan 2011;11(3):316-326.

10. Erdmann AL, Backes D, Minuzzi H. Gerência do cuidado de enfermagem pelo olhar da complexidade. Online braz.j. nurs. [Internet]. 2001[citado 1 abril 2014];7(1). Disponible en: http://www. objnursing.uff.br/index.php/nursing/article/view/j.1676-4285.2008.1033/313

11. Kérouac S. El pensamiento enfermero. $4^{\mathrm{a}}$ reimp ed. Barcelona: Masson; 2005.

12. Milos P, Larraín AL, Simonetti M. Categorización de servicios de enfermería: propuesta para asegurar una atención de calidad en tiempo de escasez de enfermeras. Cienc. enferm. 2009; XV(1):17-24.

13. Zárate Grajales RA. La gestión del cuidado de enfermería. Index Enferm 2004:4445.

14. Estefo Agüero S, Paravic Klijn T. Enfermería en el rol de gestora de los cuidados. Cienc. enferm. 2010; XVI(3):33-39.

15. Thofehrn MB, Traversi MS, Muniz RM, Duarte AC, Leite MP. O processo de enfermagem no cotidiano de acadêmicos de Enfermagem e enfermeiros. Rev Gaucha Enferm. 1999:20(1):69-79.

16. Matsuda LM, Meireles VC, Gomes FV, Salfeld SMS, Molina RM. Instrumentos administrativos: percepção de enfermeiros de um hospital universitário. Cogitare Enferm. 2010;15(1): 117-123.

17. Seguel F, Paravic T. Unidad de investigación en hospitales autogestionados. Cienc. enferm. 2011; XVII (2): 19-25.

18. Gonzáles Pérez A, Villazon Castro N, Almira Bello K, Cowley Noble A, Mugarra Romero CG. Gestión de cuidados con calidad desde la formación del profesional de enfermería. Rev Cubana Enferm. 2011;27(4):319-326.

19. Harrison L, Ray A, Cianelli R, Rivera MS, 
Urrutia M. Competencias en investigación para diferentes niveles de formación de enfermeras: una perspectiva latinoamericana. Cienc. enferm. 2005; XI (1):5971.
20. Chailán PMM, Conchall PTJ. El saber práctico en enfermería. Rev Cubana Enferm 2010;26 (2):111-117.

21. Sánchez Caro J. Configuración actual de la relación clínica. Rev Adm Sanit. 2004;2(3):433-462.

\section{ANEXO 1 \\ Cuestionario gestión del cuidado de enfermería Chile 2011}

Lea las instrucciones cuidadosamente, de antemano ¡MUCHAS GRACIAS POR SU COLABORACIÓN!

Complete o marque con una $\mathrm{X}$ la alternativa correspondiente.

Nombre de la institución

\section{DATOS SOCIODEMOGRÁFICOS}

Sexo: $\square$ Masculino $\square$ Femenino Nacionalidad:

Estado civil: $\square$ Soltero(a) $\square$ Casado(a) $\quad \square$ Pareja de Hecho

$\square$ Divorciado(a) $\square$ Viudo (a)

Edad

\section{DATOS DE FORMACIÓN ACADÉMICA}

Nivel de estudio de enfermería:

$\square$ Licenciatura

Postítulo

Institución de formación:

$\square$ Pública $\square$ Privada

Año de egreso:

Estudio de posgrado

Diplomados

Magíster

Doctorados

Cursos Generales. Si es sí, en cuál área:

¿Ha realizado algún tipo de curso o diplomado sobre gestión del cuidado de enfermería?
$\mathrm{Si}$
NO
Dónde Cuántos

\section{DATOS DE TRAYECTORIA PROFESIONAL}

Servicio en cual trabaja actualmente:

$\square$ Cirugía $\square$ Medicina $\square$ UTI $\square$ Urgencias $\quad \square$ UCI $\square$ Pensionado

$\square$ Pabellón/recuperación $\square$ Ambulatorio $\square$ otro, especifique

Cargo desempeñado actualmente en la institución:

$\square$ Enfermera clínica por turnos $\square$ Enfermera supervisora

$\square$ Enfermera clínica diurna. $\square$ Enfermeras en labor de gestión

Años de permanencia en la institución:

Mayor a 6 meses hasta 3 años.

4-7 años

$8-10$ años

Más de 10 años 


\begin{tabular}{|c|c|c|c|c|c|}
\hline 4. Respecto a la Gestión del cuidado & 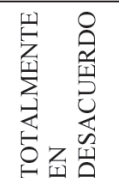 & 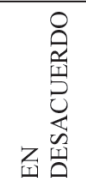 & 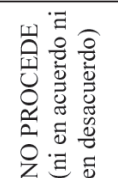 & 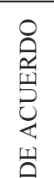 & 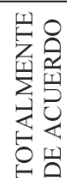 \\
\hline Puntaje & 1 & 2 & 3 & 4 & 5 \\
\hline $\begin{array}{l}4.1 \text { ¿Las unidades de gestión del cuidado facilitan la disciplina de } \\
\text { enfermería? }\end{array}$ & 1 & 2 & 3 & 4 & 5 \\
\hline $\begin{array}{l}4.2 \text { ¿Considera que la gestión del cuidado trae consigo implicancias } \\
\text { ético-legales para la enfermería? }\end{array}$ & 1 & 2 & 3 & 4 & 5 \\
\hline 4.3 ¿Conoce Ud. las normativas relacionadas a la gestión del cuidado? & 1 & 2 & 3 & 4 & 5 \\
\hline 5. Respecto a la gestión del cuidado, ¿cómo la considera Ud.? & 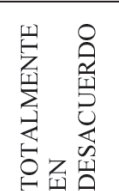 & 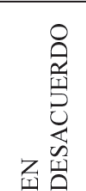 & 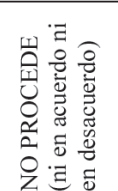 & 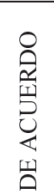 & 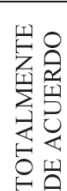 \\
\hline $\begin{array}{l}\text { 5.1. Una actividad realizada solo del(la) gestor(a) del cuidado, por lo } \\
\text { tanto no puedo realizar GC desde mi puesto de trabajo. }\end{array}$ & 1 & 2 & 3 & 4 & 5 \\
\hline 5.2. Una actividad realizada por las enfermeras en su conjunto. & 1 & 2 & 3 & 4 & 5 \\
\hline $\begin{array}{l}\text { 5.3. Una actividad de trabajo en conjunto (gestor(a) y equipo de } \\
\text { enfermería) }\end{array}$ & 1 & 2 & 3 & 4 & 5 \\
\hline 5.4. Otro profesional puede también realizar gestión del cuidado. & 1 & 2 & 3 & 4 & 5 \\
\hline 6. La actividad principal de gestión del cuidado es: & 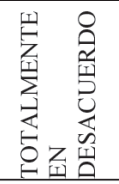 & 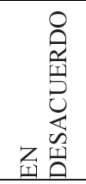 & 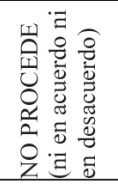 & 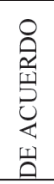 & 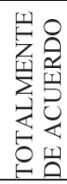 \\
\hline $\begin{array}{l}\text { 6.1. Planificar, organización, dirección y control de los cuidados } \\
\text { integrales de enfermería }\end{array}$ & 1 & 2 & 3 & 4 & 5 \\
\hline 6.2. Diseñar, realizar y evaluar protocolos o guías clínicas & 1 & 2 & 3 & 4 & 5 \\
\hline $\begin{array}{l}\text { 6.3. Corresponde a la instauración de un modelo teórico (teorías de } \\
\text { enfermería) para las actividades diarias. }\end{array}$ & 1 & 2 & 3 & 4 & 5 \\
\hline 6.4. Facilitar el empoderamiento de la enfermería en la institución & 1 & 2 & 3 & 4 & 5 \\
\hline $\begin{array}{l}\text { 7. ¿Cómo conceptualiza o concibe Ud. la gestión del cuidado en su } \\
\text { unidad? }\end{array}$ & 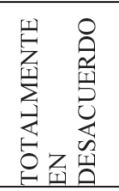 & 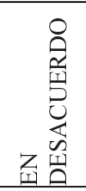 & 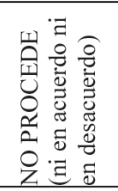 & 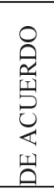 & 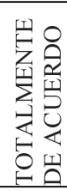 \\
\hline $\begin{array}{l}\text { 7.1. Como una actividad solo le corresponde al gestor(a) del cuidado, } \\
\text { por lo tanto es imposible realizar la gestión del cuidado desde mi puesto } \\
\text { de trabajo. }\end{array}$ & 1 & 2 & 3 & 4 & 5 \\
\hline $\begin{array}{l}\text { 7.2. Esta se realiza siguiendo los lineamientos impartidos desde la } \\
\text { unidad de gestión del cuidado, facilitando la retroalimentación de la } \\
\text { actividad clínica asistencial. }\end{array}$ & 1 & 2 & 3 & 4 & 5 \\
\hline $\begin{array}{l}\text { 7.3. Como una actividad global desde el gestor(a) del cuidado hacia las } \\
\text { bases (enfermeras clínicas), con el fin de brindar un servicio de calidad } \\
\text { al usuario. }\end{array}$ & 1 & 2 & 3 & 4 & 5 \\
\hline 8.¿Cómo implementa Ud. la gestión del cuidado en su unidad? & 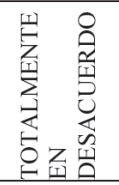 & 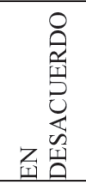 & 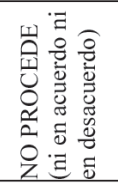 & 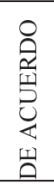 & 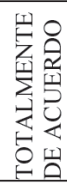 \\
\hline $\begin{array}{l}\text { 8.1. Poniendo en marcha protocolos, registros y notas de enfermería de } \\
\text { la institución. }\end{array}$ & 1 & 2 & 3 & 4 & 5 \\
\hline $\begin{array}{l}\text { 8.2. Participando activamente en las actividades de enfermería, } \\
\text { utilizando como base la teorías propuestas (investigación, docencia) }\end{array}$ & 1 & 2 & 3 & 4 & 5 \\
\hline $\begin{array}{l}\text { 8.3. Facilitando la retroalimentación entre la actividad clínica y la } \\
\text { supervisión de enfermería. }\end{array}$ & 1 & 2 & 3 & 4 & 5 \\
\hline
\end{tabular}




\begin{tabular}{|c|c|c|c|c|c|}
\hline 9¿Qué ámbitos considera Ud. que comprende la gestión del cuidado? & 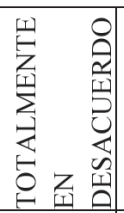 & 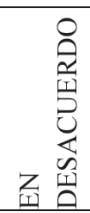 & 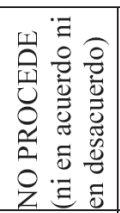 & 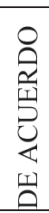 & 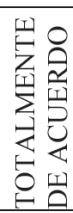 \\
\hline 9.1. Ámbito Clínico/asistencial & 1 & 2 & 3 & 4 & 5 \\
\hline 9.2. Ámbito Administrativo & 1 & 2 & 3 & 4 & 5 \\
\hline 9.3. Ámbito Docente/investigación. & 1 & 2 & 3 & 4 & 5 \\
\hline 9.4. Ámbito Gerencial & 1 & 2 & 3 & 4 & 5 \\
\hline 9.5. Ámbito Legal & 1 & 2 & 3 & 4 & 5 \\
\hline $\begin{array}{l}10 \text { ¿Qué elementos considera Ud. como pilar fundamental para que } \\
\text { se lleve a cabo en su unidad? }\end{array}$ & 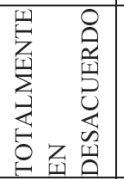 & 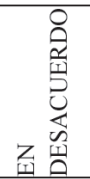 & 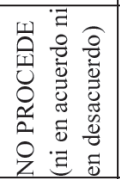 & 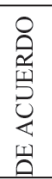 & 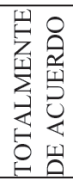 \\
\hline 10.1. Investigación en enfermería. & 1 & 2 & 3 & 4 & 5 \\
\hline $\begin{array}{l}\text { 10.2. Formación continua sobre gestión del cuidado a los enfermeros(as) } \\
\text { en la institución. }\end{array}$ & 1 & 2 & 3 & 4 & 5 \\
\hline 10.3. Formación académica de gestión del cuidado desde pregrado. & 1 & 2 & 3 & 4 & 5 \\
\hline $\begin{array}{l}\text { 10.4. Participación activa de los enfermeros en la atención integral al } \\
\text { usuario, gestionar los cuidados integrales a cada usuario. }\end{array}$ & 1 & 2 & 3 & 4 & 5 \\
\hline $\begin{array}{l}\text { 10.5. Reconocimiento de la institución a la gestión del cuidado de } \\
\text { enfermería }\end{array}$ & 1 & 2 & 3 & 4 & 5 \\
\hline
\end{tabular}

¡MUCHAS GRACIAS POR SU COLABORACIÓN! 